University of Nebraska - Lincoln

DigitalCommons@University of Nebraska - Lincoln

Robert G. Fuller Publications and Presentations Research Papers in Physics and Astronomy

August 1970

\title{
TRANSIENT OPTICAL ABSORPTION BY SELF-TRAPPED EXCITONS IN ALKALI HALIDE CRYSTALS
}

Robert Fuller

rfuller@neb.rr.com

R.T. Williams

Naval Research Laboratory, Washington, D. C.

M.N. Kabler

Naval Research Laboratory, Washington, D. C.

Follow this and additional works at: https://digitalcommons.unl.edu/physicsfuller

Part of the Physics Commons

Fuller, Robert; Williams, R.T.; and Kabler, M.N., "TRANSIENT OPTICAL ABSORPTION BY SELF-TRAPPED EXCITONS IN ALKALI HALIDE CRYSTALS" (1970). Robert G. Fuller Publications and Presentations. 14.

https://digitalcommons.unl.edu/physicsfuller/14

This Article is brought to you for free and open access by the Research Papers in Physics and Astronomy at DigitalCommons@University of Nebraska - Lincoln. It has been accepted for inclusion in Robert G. Fuller Publications and Presentations by an authorized administrator of DigitalCommons@University of Nebraska - Lincoln. 
exists between maximum eigenvalue and eigenvector of the transfer matrix for the ferroelectric two-dimensional model ${ }^{15}$ and the groundstate eigenvector for the one-dimensional anisotropic Heisenberg chain.

It appears from our work that the transverse field changes the critical temperature without changing the critical behavior until the critical field is reached, when there is a sudden change in the critical behavior at $T=0$ which becomes like the critical behavior of the Ising system in one higher dimension.

\footnotetext{
*Work supported by Centre National de la Recherche Scientifique. On leave of absence from Laboratoire de Physique des Solides, Faculté des Sciences, Paris $V^{e}$, France.
}

\author{
${ }^{1}$ P. G. de Gennes, Solid State Commun. 1, 132 (1963). \\ ${ }^{2}$ Y. L. Wang and B. Cooper, Phys. Rev. $\underline{172}, 539$ \\ (1968). \\ ${ }^{3} \mathrm{~J}$. Ginibre, to be published. \\ ${ }^{4}$ R. J. Elliott and P. Pfeuty, to be published. \\ ${ }^{5}$ H. B. Callen, Phys. Rev. 130, 890 (1963). \\ ${ }^{6} \mathrm{C}$. Wood and R. J. Elliott, to be published. \\ ${ }^{7}$ T. Matsubara, Progr. Theor. Phys. 14, 351 (1955). \\ ${ }^{8}$ M. E. Fisher, Rep. Progr. Phys, 30, 615 (1967). \\ ${ }^{9}$ P. Pfeuty, Ann. Phys. (New York) 57, $79(1970)$. \\ ${ }^{10} \mathrm{E}$. Lieb, T. Schultz, and D. Mattis, Ann. Phys. \\ (New York) 16, 407 (1961). \\ ${ }^{11} \mathrm{P}$. Pfeuty and R. J. Elliott, to be published. \\ ${ }^{12}$ L. P. Kadanoff et al., Rev. Mod. Phys. 39,395 \\ (1967). \\ ${ }^{13}$ H. Falk and L. Bruch, Phys. Rev. 180, 442 (1969). \\ ${ }^{14}$ T. D. Schultz, D. C. Mattis, and E. Lieb, Rev. \\ Mod. Phys. 36, 856 (1967). \\ ${ }^{15} \mathrm{E}$. Lieb, Phys. Rev. Lett. 19, 108 (1967). \\ ${ }^{16}$ M. E. Fisher and D. S. Gaunt, Phys. Rev. 133, 224 \\ (1964).
}

\title{
TRANSIENT OPTICAL ABSORPTION BY SELF-TRAPPED EXCITONS IN ALKALI HALIDE CRYSTALS
}

\author{
R. G. Fuller,* R. T. Williams, and M. N. Kabler \\ Naval Research Laboratory, Washington, D. C. 20390
}

(Received 29 June 1970)

\begin{abstract}
Transient absorption spectra produced by pulsed electron excitation at low temperature have been identified as due to transitions originating in the lowest triplet states of the self-trapped exciton. The current model, comprising an electron plus a self-trapped hole, is shown to provide electronic configurations for the higher excited states which give a good account of the principal features of the spectra.
\end{abstract}

Exciton self-trapping in simple halide lattices is an established phenomenon. It gives rise to broad-band, strongly Stokes-shifted luminescence which has been studied in alkali, ${ }^{1}$ ammonium, ${ }^{2}$ and alkaline-earth ${ }^{3}$ halides. The selftrapping can be attributed to the formation of a covalent bond between two adjacent excited halide ions, and the resulting $X_{2}^{-2}$ molecular ion provides metastable singlet and triplet states with which the characteristic short- and longlived luminescent transitions can be reasonably well explained. ${ }^{4}$ In pure materials at low temperatures, a significant fraction of any energy imparted to the electronic system is at some stage stored in these states. The present work concerns absorption spectra arising from the longerlived states of self-trapped excitons in several alkali halides. The model with which the luminescent transitions have been interpreted will be shown to furnish also a straightforward account of the higher excited states involved in the absorption.
The basic experiment consisted of time-resolved measurements of absorption and emission spectra produced by single-pulse excitation from an electron source of $500 \mathrm{keV}$ mean energy. The apparatus has been described previously. ${ }^{5} \mathrm{~A}$ novel aspect of this system is the use of a light beam which reflects internally at a low angle from the crystal face being irradiated. This geometry maximizes the light path through the irradiated volume, which is thin $(\approx 0.5 \mathrm{~mm})$ because of the low penetration of the electron beam. For more accurate spectral resolution, the system was augmented by a simple rotating-mirror scanning device capable of sweeping at rates up to $6 \mathrm{~nm} / \mu \mathrm{sec}$. Repetitive excitation and a digital signal averager were used when it was desired to minimize the intensity of a given pulse in order to hold transient heating effects to tolerable level. ${ }^{6}$ The decay times under investigation fell in the $10^{-6}$ - to $10^{-2}$ - sec range. No attempt was made to observe the singlet self-trapped exciton states since their lifetimes (1-10 nsec) are only 


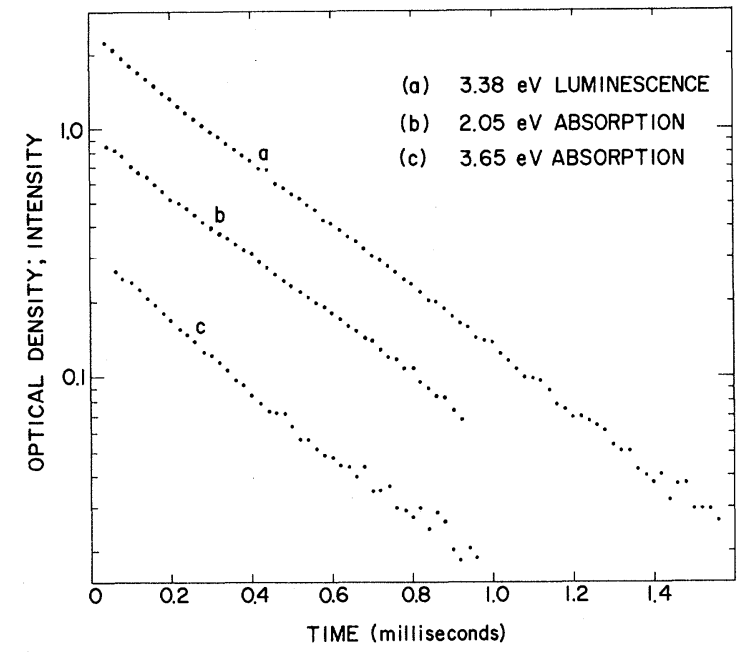

FIG. 1. Decay of optical density of absorption and intensity of intrinsic luminescence in $\mathrm{NaCl}$ at about $8^{\circ} \mathrm{K}$. With the exception of a small permanent $F$ band, all portions of the observed absorption spectrum decay with the same lifetime as the two bands shown.

on the verge of accessibility with this apparatus.

Observed absorption was identified as due to self-trapped excitons principally on the basis of decay-time correlations with the luminescence. A typical example is shown in Fig. 1, where the decay of the 3.38-eV triplet-state emission band in $\mathrm{NaCl}$ is compared with the decays of two prominent new absorption bands. The sample temperature was $8 \pm 2^{\circ} \mathrm{K}$. Each point represents a separate channel of the signal averager, ten runs having been averaged for the absorption data in this example. Within the experimental accuracy, the three curves are parallel and exhibit identical lifetimes of $3.4 \times 10^{-4}$ sec. $^{7}$ This time correlation persists well into the temperature range where the lifetimes begin to decrease markedly because of thermally activated nonradiative transitions. ${ }^{8}$ These facts lead us to infer that the lowest triplet self-trapped-exciton state is the origin of the absorption at these wavelengths.

Figure 2 shows transient absorption spectra for three alkali chlorides at $8 \pm 2^{\circ} \mathrm{K}$. Each solid curve represents that part of the initial absorption which decays in proportion to the intensity of the triplet-state luminescence. The electron pulse also creates transient and stable $F, H$, and $V_{k}$ centers which contribute to the absorption. In $\mathrm{KCl}$, for example, $F$ and $H$ centers give strong nonexponential components of roughly $15 \mu \mathrm{sec}$ duration. The large time difference between these and the 5-msec self-trapped-exciton absorption makes their separation straightforward. Fast transients in the $F$ and $H$ bands were first

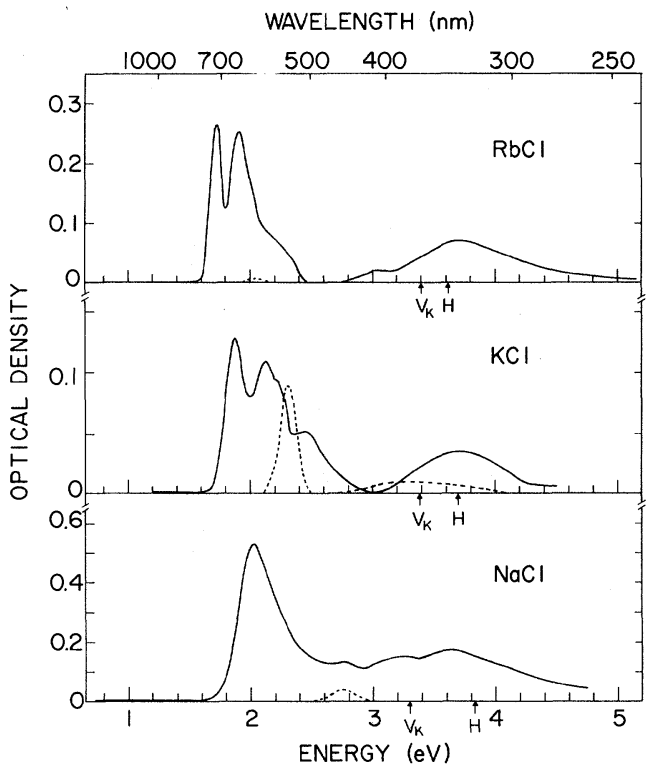

FIG. 2. Absorption spectra of self-trapped excitons (solid lines) and stable defects (dashed lines) produced at low temperature by single electron pulses. The pulse energy was comparable in the three cases. Peak wavelengths of $\boldsymbol{H}$ and $V_{k}$ bands are marked for reference.

observed at $10^{\circ} \mathrm{K}$ by Kondo et al. and were attributed to mutual annihilation of $F$ and $H$ centers. ${ }^{9}$

The approximate amounts of stable color-center absorption produced by one pulse are shown as the dashed bands in Fig. 2. Although the Fband corrections were fairly clear from the data, inflections in the solid curves near the peaks of the $F$ bands for $\mathrm{KCl}$ and $\mathrm{NaCl}$ are nevertheless subject to some uncertainty. Additional measurements imply that the appearance of stable $V_{k}$ centers in the spectrum for $\mathrm{KCl}$ is correlated with the relatively large concentration of $F$ centers, which act as electron traps and thereby stabilize the $V_{k}$ centers. As is the case for the luminescence, trace impurities or accumulated radiation damage appear to have no significant influence on the absorption spectra. The $\mathrm{KCl}$ spectrum of Fig. 2, for example, comes from an ultrapure, zone-refined crystal, ${ }^{10}$ but spectra from Harshaw crystals were similar.

New transient absorptions correlated with the luminescent triplet states have also been observed in $\mathrm{KBr}, \mathrm{RbBr}, \mathrm{KI}$, and RbI. Spectral measurements have been completed only on the two bromides, whose spectra strongly resemble that of $\mathrm{KCl}$ in Fig. 2. Peak energies are given in Table I for the three most prominent bands. Only two bands are noted for $\mathrm{NaCl}$, for which the 
Table I. Peak energies (eV) of principal self-trappedexciton absorption bands: (a) the first two peaks of the red-infrared series, (b) the broad ultraviolet band.

\begin{tabular}{|c|c|c|c|}
\hline & \multicolumn{2}{|c|}{ (a) } & (b) \\
\hline $\mathrm{RbCl}$ & 1.72 & 1.90 & 3.7 \\
\hline $\mathrm{KCl}$ & 1.87 & 2.12 & 3.7 \\
\hline $\mathrm{NaCl}$ & \multicolumn{2}{|c|}{2.03} & 3.6 \\
\hline $\mathrm{RbBr}$ & 1.48 & 1.65 & 3.35 \\
\hline $\mathrm{KBr}$ & 1.58 & 1.77 & 3.30 \\
\hline
\end{tabular}

doublet structure is the red band is not resolved.

Let us now consider the electronic states which give rise to these optical transitions. The luminescence data have shown that the ionized state of the self-trapped exciton is in fact the selftrapped hole, or $V_{k}$ center. This center is basically an $X_{2}{ }^{-}$molecular ion and, in the chlorides, its predominant absorption band at approximately $3.4 \mathrm{eV}$ has been identified as a $\left(\sigma_{g} 3 p\right)\left(\pi_{u} 3 p\right)^{4}$ $\times\left(\pi_{g} 3 p\right)^{4}\left(\sigma_{u} 3 p\right)^{2},{ }^{2} \Sigma_{g}{ }^{+}-\left(\sigma_{g} 3 p\right)^{2}\left(\pi_{u} 3 p\right)^{4}\left(\pi_{g} 3 p\right)^{4}\left(\sigma_{u} 3 p\right)$, ${ }^{2} \Sigma_{u}{ }^{+}$transition. ${ }^{11,12}$ We designate these configurations $B$ and $A$, respectively. For the lowest state of the exciton, it is reasonable to assign the electron to a $\sigma_{g} 4 s$ orbital having substantial amplitude on the ions neighboring the $\mathrm{Cl}_{2}{ }^{-} \cdot{ }^{4} V_{k}$ like absorption should therefore persist in the exciton, and consequently we attribute the absorption in the $3-$ to $4-\mathrm{eV}$ range to this type of hole transition. The peak is $0.3 \mathrm{eV}$ above the known $V_{k}$ peak energy in all three chlorides. Regarding the absorption in the $1-$ to $3-\mathrm{eV}$ range, the logical source is a sequence of Rydberg-like transitions converging to the conduction band edge. The electron orbitals involved would be $1 s \sigma_{g}, 2 p \sigma_{u}, 2 p \pi_{u}$, etc., employing an eclectic but convenient notation. It is apparent in Fig. 2 that the shifts in this absorption from crystal to crystal are similar to the shifts for electron-excess color centers, in particular $F$ and $M$ centers.

Energy-level assignments based on the present considerations are shown for the three chlorides in Fig. 3. Selection rules on multiplicity and parity have been taken into account. The lowest triplet state is placed at zero energy since it originates all transitions under consideration. Figure 3 pertains only to lattice configurations characteristic of the ${ }^{3} \Sigma_{u}{ }^{+}$state; that is, FranckCondon transitions are assumed. The free-exciton state represents an exciton which has escaped the trapping site; the energy of this state relative to the ${ }^{1} \Sigma_{g}{ }^{+}$ground state is just the peak energy of the first exciton absorption band in the perfect crystal. From the location of the free-

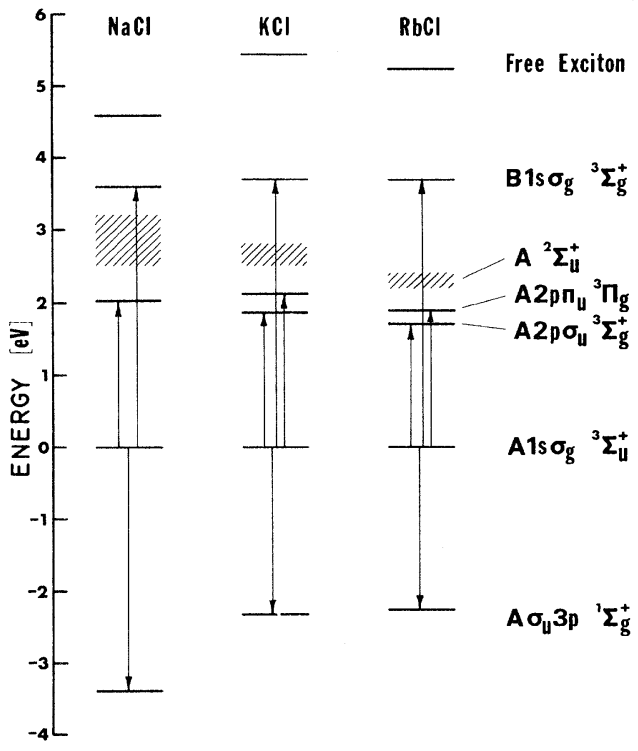

FIG.3. Energy level diagram for principal transitions originating in the lowest ${ }^{3} \Sigma_{u}{ }^{+}$state of the selftrapped exciton in three alkali chlorides. From the measured spectra, the conduction-band edge is estimated to be within the range indicated by the crosshatching.

exciton state it is clear that none of the observed transitions involve states degenerate with exciton band states.

The conduction band edge or Rydberg series limit, $A^{2} \Sigma_{u}{ }^{+}$, cannot be located accurately on the basis of the spectra in Fig. 2. Only a rough estimate has been made, partly by comparison with Rydberg-like color-center absorptions in these crystals. ${ }^{13}$ This results in placement of the conduction-band edges within the ranges of uncertainty indicated by the cross hatchings in Fig. 3 . It thus becomes evident that the optical ionization energies of self-trapped excitons are substantially greater than the $0.5-$ to $1.0-\mathrm{eV}$ free-exciton binding energies inferred from various fundamental spectra. ${ }^{14}$ It is apparent also that the $B 1 s \sigma_{g}{ }^{3} \Sigma_{g}{ }^{+}$states are degenerate with the conduction bands.

There are two possible interpretations for the doublet structures in the $1.5-$ to $2.1-\mathrm{eV}$ range in $\mathrm{KCl}$ and $\mathrm{RbCl}$ (and also $\mathrm{KBr}$ and $\mathrm{RbBr}$ ): transitions to $2 p$ - and $3 p$-like orbitals or to molecularfield-split $2 p \sigma_{u}$ and $2 p \pi_{u}$ orbitals. The relative strengths seem to favor the latter assignment, and the designations in Fig. 3 reflect this choice. Note in Table I that the doublet splittings are about $0.2 \mathrm{eV}$ in both the chlorides and the bromides.

In light of the present data, it is useful to com- 
pare the self-trapped exciton with a well-known color center, the $M$ center. With respect to the character of the excited electron orbital, the transitions which give rise to the $M_{1}$ and $M_{2}$ absorption bands are similar to our proposed molecular-field-split Rydberg transitions; in fact, for the crystals in Table I, the exciton bands comprising the red doublet fall consistently between the $M_{1}$ and $M_{2}$ bands. ${ }^{15}$ Thus the transitions appear relatively insensitive to whether the electron is interacting with an $X_{2}{ }^{-}$molecular ion, as for the exciton, or with another electron in the vacancy pair, as for the $M$ center. This suggests a significant degree of delocalization for the $1 s \sigma_{g}, 2 p \sigma_{u}$, and higher orbitals of the Rydberg series. It may be noted that the lifetime data also relate to the question of delocalization. After correction for multiplicity forbiddenness, the dipole matrix elements for ${ }^{1} \Sigma_{u}{ }^{+} \rightarrow{ }^{1} \Sigma_{g}{ }^{+}$transitions ${ }^{4}$ still exceed those for the ${ }^{3} \Sigma_{u}{ }^{+}{ }^{1}{ }^{1} \Sigma_{g}{ }^{+}$transitions by factors ranging from roughly 3 for $\mathrm{NaCl}$ to 6 for $\mathrm{RbBr} .{ }^{16}$ It is reasonable to attribute this effect primarily to a relatively greater radial extent of the $1 s \sigma_{g}$ orbital in the ${ }^{3} \Sigma_{u}{ }^{+}$state. Theoretical indications of diffuse relaxed excitation states have previously appeared in the work of Wood. ${ }^{17}$

A more extensive account of the present work will be published.

\footnotetext{
* Now at the University of Nebraska, Lincoln, Neb.

${ }^{1}$ See, for example, M. N. Kabler, Phys. Rev. 136, A1296 (1964); R. B. Murray and F。J. Keller, Phys。 Rev. 137, A942 (1965); J. Ramamurti and K. Teegarden, Phys. Rev. 145, 698 (1966); M. Ikezawa and T. Kojima, J. Phys. Soc.Jap. 27, 1551 (1969), and references therein.

${ }^{2}$ M. J. Marrone and M. N. Kabler, Phys. Rev. $\underline{176}$, 1070 (1968).

${ }^{3}$ W. Hayes, D. L. Kirk, and G. P. Summers, Solid State Commun. 7, 1061 (1969).

${ }^{4}$ M. N. Kabler and D. A. Patterson, Phys. Rev. Lett. 19,652 (1967).

${ }^{5}$ R. T. Williams, R. G. Fuller, M. N. Kabler, and V.H. Ritz, Rev。Sci.Instrum. 40, 1361 (1969).
}

${ }^{6} \mathrm{In} \mathrm{KCl}$ and $\mathrm{RbCl}$ normal excitation pulses produced sufficient heating to cause fast transients in the decays. Only these two crystals were affected, evidently because their thermal quench temperatures $\left(\approx 12^{\circ} \mathrm{K}\right)$ are lower than those for the other crystals by at least a factor of 3. It proved possible to work with pulse energies low enough to eliminate these transients. The pulses ranged in energy density from 0.004 to $0.02 \mathrm{~J} / \mathrm{cm}^{2}$ and were roughly $5 \mathrm{nsec}$ in duration. Rise times for absorption and luminescence were less than the time resolution, roughly 20 nsec.

${ }^{7}$ This lifetime is roughly $10 \%$ larger than that reported in Ref. 4. The origin of the discrepancy is not understood. The present data are reproducible and are considered the more reliable.

${ }^{8}$ If a given state originates both absorption and emission and if the radiative transition probability is independent of temperature, then the absorption will be proportional to the emission intensity. In $\mathrm{RbCl}$, uniquely among the crystals investigated, the decay was slightly nonexponential even for the lowest intensities of excitation. Tangents to the curve gave lifetimes ranging from 5 to $10 \mathrm{msec}$. The absorption was nevertheless also proportional to the emission intensity。

${ }^{9} \mathrm{Y}$. Kondo, M. Hirai, T. Yoshinari, and M. Ueta, J. Phys. Soc. Jap. 26, 1553 (1969)。

${ }^{10}$ This $\mathrm{KCl}$ crystal was kindly provided by C. T. Butler, Solid State Division, Oak Ridge National Laboratory, Oak Ridge, Tenn.

${ }^{11}$ C. J. Delbecq, W. Hayes, and P. H. Yuster, Phys. Rev. 121, 1043 (1961).

${ }^{12}$ For the present discussion, designation of states in terms of the point group of a homonuclear diatomic molecule $D_{\infty h}$ is adequate and convenient. In the $\mathrm{NaCl}$ structure the actual group is $D_{2 h}$.

${ }^{13}$ For this purpose the $K$ band of the $F$ center has been given primary consideration. See G. Spinole and D. Y. Smith, Phys. Rev. 140, A2117, A2121 (1965).

${ }^{14}$ See, for example, D. Fröhlich and B. Staginnus, Phys. Rev. Lett. 19, 496 (1967).

${ }^{15}$ W. D. Compton and H. Rabin, in Solid State Physics, edited by $\mathrm{H}$. Ehrenreich, F. Seitz, and D. Turnbull (Academic, New York, 1964), Vol. 16, p. 121. In KCl, for example, the $M_{1}$ band is at $1.55 \mathrm{eV}$ and the $M_{2}$ at $2.3 \mathrm{eV}$.

${ }^{16}$ See Ref. 4 . The present computations use the ${ }^{1} \Sigma_{u}^{+} \rightarrow{ }^{1} \Sigma_{g}^{+}$lifetimes measured by I. M. Blair, D. Pooley, and D. Smith, J。Lumin. 1, 324 (1970)。

${ }^{17}$ R. F. Wood, Phys. Rev. 151, 629 (1966). 